\title{
Short communication: Signs of host genetic regulation in the microbiome composition in 2 dairy breeds: Holstein and Brown Swiss
}

\author{
O. Gonzalez-Recio,${ }^{*} \dagger^{1}$ I. Zubiria, $\ddagger$ A. García-Rodríguez, $\ddagger$ A. Hurtado,§ and R. Atxaerandio $\ddagger$ \\ *Departamento de Mejora Genética Animal, Instituto Nacional de Investigación y Tecnología Agraria y Alimentaria, 28040 Madrid, Spain \\ †Departamento de Produccion Agraria, Escuela Técnica Superior de Ingeniería Agronómica, Alimentaria y de Biosistemas, \\ Universidad Politécnica de Madrid, Ciudad Universitaria s/n, 28040 Madrid, Spain \\ ‡Departamento de Producción Animal, NEIKER-Tecnalia, Granja Modelo de Arkaute Apartado 46, 01080 Vitoria-Gasteiz, Spain \\ §Departamento de Sanidad Animal, NEIKER-Tecnalia, Berreaga 1, 48160 Derio, Spain
}

\section{ABSTRACT}

This study aimed to evaluate whether the host genotype exerts any genetic control on the microbiome composition of the rumen in cattle. Microbial DNA was extracted from 18 samples of ruminal content from 2 breeds (Holstein and Brown Swiss). Reads were processed using mothur (https://www.mothur.org/) in $16 \mathrm{~S}$ and $18 \mathrm{~S}$ rRNA gene-based analyses. Then, reads were classified at the genus clade, resulting in 3,579 operational taxonomic units (OTU) aligned against the $16 \mathrm{~S}$ database and $184 \mathrm{OTU}$ aligned against the $18 \mathrm{~S}$ database. After filtering on relative abundance $(>0.1 \%)$ and penetrance (95\%), 25 OTU were selected for the analyses (17 bacteria, 1 archaea, and 7 ciliates). Association with the genetic background of the host animal based on the principal components of a genomic relationship matrix based on single nucleotide polymorphism markers was analyzed using Bayesian methods. Fifty percent of the bacteria and archaea genera were associated with the host genetic background, including Butyrivibrio, Prevotella, Paraprevotella, and Methanobrevibacter as main genera. Forty-three percent of the ciliates analyzed were also associated with the genetic background of the host. In total, $48 \%$ of microbes were associated with the host genetic background. The results in this study support the hypothesis and provide some evidence that there exists a host genetic component in cattle that can partially regulate the composition of the microbiome.

Key words: genomic, Holstein, microbiome, metagenome

\section{Short Communication}

Research interest in the microbiome and its effects on complex traits in both humans (Huttenhower et

Received May 17, 2017.

Accepted November 1, 2017.

${ }^{1}$ Corresponding author: gonzalez.oscar@inia.es al., 2014; Waldor et al., 2015) and livestock (Jewell et al., 2015; Malmuthuge and Guan, 2016) has increased recently. The microbiome plays an important role in the phenotypic expression of many traits such as feed efficiency, disease status, and methane emission (Zhang et al., 2007). Traditionally, microbes have been studied in the laboratory without considering the interaction with the host or how they can modulate the expression of complex traits such as gut metabolism or disease incidence. In the particular case of livestock, the traits of interest are usually related to productive, health, or environmental factors. In the last decade, more attention has been placed on the interactions between microbes and diets (Saro et al., 2012, 2014; Mohammed et al., 2014; Henderson et al., 2015), methane emissions (Deng et al., 2008; Yáñez-Ruiz et al., 2010; Hayes et al., 2013; Wallace et al., 2015; Wadhwa et al., 2016), and the microbiome composition across hosts, environment, and age (Henderson et al., 2015; Jewell et al., 2015; Wang et al., 2016). The microbiome has also been proposed as a predictor of complex traits (Ross et al., 2013; Wallace et al., 2015).

Therefore, there is interest in determining whether a host genetic control exists that determines the microbiome composition. Recent studies show some evidence that supports the hypothesis that there is some sort of host control over the composition of the microbiome in mammals. For instance, Weimer et al. (2010) reported that after a near-total exchange of ruminal contents, the ruminal bacterial composition returned to a status similar to that prior to the exchange. More recently, Roehe et al. (2016) showed differences between sire progeny groups on the archaea:bacteria ratio in Aberdeen Angus and Limousin cattle breeds, and Goodrich et al. (2016) reported heritabilities greater than 0.20 for the relative abundance (RA) of several microbes in a twin human study.

The host genetic control of the ruminal microbiome composition could be used in breeding programs to select individuals with a favorable microbiome compo- 
sition for a given breeding goal, such as the reduction of enteric methane yield or the improvement of feed efficiency. The objective of this study was to determine whether genetic differences between Holstein and Brown Swiss cows exist for the RA of the rumen microbiome.

This trial was carried out in accordance with Spanish Royal Decree 53/2013 for the protection of animals used for experimental and other scientific purposes. In this study, ruminal content was sampled from 18 dairy cows (10 Holstein and 8 Brown Swiss) from Fraisoro Farm School (Zizurkil, Gipuzkoa, Spain). These cows were undergoing a nutrition experiment. They were randomly assigned to 1 of 2 concentrate supplement treatments. Both breeds were fed both diets in a balanced design in which half the cows from each breed were assigned to each treatment. Concentrates were formulated to contain cold-pressed rapeseed cake or palm as fat sources and to provide equal amounts of $\mathrm{CP}$, energy, and fat. Details on the feed formulation are provided in Supplemental Table S1 (https://doi.org/10 .3168/jds.2017-13179).

Rumen content was sampled 4 times within $48 \mathrm{~h}$ from each cow to cover the whole circadian cycle $(0000 \mathrm{~h}$ on $\mathrm{d} 1,1200 \mathrm{~h}$ on d 1, $0600 \mathrm{~h}$ on d 2, and $1800 \mathrm{~h}$ on d 2). Ruminal samples were collected from each dairy cow using a stomach tube connected to a mechanical pumping unit. About $100 \mathrm{~mL}$ of ruminal extraction from each of the 4 time points was placed in a unique container per animal to obtain a single mixed sample per animal. All samples were frozen immediately after the extraction and then stored at $-20 \pm 5^{\circ} \mathrm{C}$ until analysis.

Then, all samples were gradually thawed overnight at refrigeration temperature $\left(5 \pm 3^{\circ} \mathrm{C}\right)$, mixed, and squeezed through 4 layers of sterile cheesecloth to separate solid (solids with a particle size smaller than the diameter of the tube) from liquid digesta phases. This latter phase was subsequently separated into planktonic organisms and bacteria associated with the liquid fraction. The solid phase was separated into associated and adherent fractions. Fractionation procedures were carried out following the methodology described in $\mathrm{Yu}$ and Foster (2005). The 4 fractions were lyophilized and composited to obtain a unique sample with the 4 fractions represented proportionally (on a DM basis).

After composition, DNA extraction was performed using the commercial Power Soil DNA isolation kit (Mo Bio Laboratories Inc., Carlsbad, CA) following the manufacturer's instructions. The extracted DNA was subjected to paired-end Illumina sequencing of the V4 hypervariable region of the 16S rRNA (Caporaso et al., 2011) and of the V7 region of the $18 \mathrm{~S}$ rRNA genes. The libraries were generated by means of a Nextera kit (Illumina Inc., San Diego, CA). The 250-bp pairedend sequencing reactions were carried out on a MiSeq platform (Illumina Inc.).

Sequence data were processed using mothur version 1.38.1.1 (Schloss et al., 2009; Kozich et al., 2013). Sequences below 220 bp in length and Phred score below 20 were discarded. After joining forward and backward reads, 3,261,168 and 3,431,242 reads remained for the analysis from the $16 \mathrm{~S}$ and $18 \mathrm{~S}$ rRNA regions, respectively. Chimeras and unique sequences were removed. Sequences were then preclustered and finally classified using the default method (Wang et al., 2007) on classify.seqs() using the GreenGenes database (May 2013 version; http://greengenes.secondgenome.com/) for the bacterial and archaeal 16S rRNA genes, whereas protozoal and fungi $18 \mathrm{~S}$ rRNA genes were aligned against the 18S SILVA database (March 2015 version; https:// www.arb-silva.de/). The reads clustered to 3,579 operational taxonomic units (OTU) after being aligned against the $16 \mathrm{~S}$ database and to $184 \mathrm{OTU}$ against the 18 S SILVA database. Data were summarized at the genus level, with 287 known genera for 16S (Supplemental Table S2; https://doi.org/10.3168/jds.2017-13179) and 49 genera for fungi and protozoa (Supplemental Table S3; https://doi.org/10.3168/jds.2017-13179). The RA of genera in each animal was calculated after excluding those genera that appeared in $<0.1 \%$ proportion in both breeds and in at least 17 animals. In total, 18 bacteria and archaea genera and 7 ciliate genera were kept for final analyses.

Genotypes from animals under study were also obtained with the Illumina 9K chip (Illumina Inc.). A total of 9,146 SNP with call rate $>95 \%$ and minor allele frequency $>0.05$ in the whole genotyped Spanish population were kept (data from more than 3,000 individuals provided by the Spanish Holstein association CONAFE; Madrid, Spain). Less than $2 \%$ of SNP were not under Hardy-Weinberg equilibrium but still remained in the analyses.

The background genetic effect was analyzed for each of the ruminal microbes selected with their RA as a dependent covariate (phenotype). A logarithmic transformation $[1+\ln (\mathrm{x})]$ was applied if the phenotypic distribution of microbial RA did not visually approximate to a Gaussian distribution. The statistical model was adjusted by diet treatment (2 groups: with or without cold-pressed rapeseed cake), age (primiparous or multiparous) groups, and DIM as a covariate. It also included the first 2 principal components $(\mathbf{P C})$ of a genomic relationship matrix that contains the genomic relationship between individuals $i$ and $j$ (Yang et al., 2011), which was calculated as 


$$
\mathbf{G}_{i j}=\frac{1}{L} \sum_{k=1}^{L} \frac{\left(g_{i k}-\hat{p}_{k}\right)\left(g_{j k}-\hat{p}_{k}\right)}{\hat{p}_{k}\left(1-\hat{p}_{k}\right)},
$$

where $g_{i k}$ refers to the gene frequency value genotypes $A A, A a$, and $a a$, coded as $1,0.5$, and 0 , respectively, of individual $i$ or $j$ at locus $k(k=1, \ldots, \mathrm{L})$, with $L$ being the number of SNP loci $(9,146)$. Gene frequency is half the number of copies of the reference allele $A$. Then, $\hat{p}_{k}$ was the estimated allele frequency in the whole genotyped population as provided by the Spanish Holstein association CONAFE.

The final model can be expressed as

$$
\begin{aligned}
R A_{i j k l m n}=\mu & + \text { diet }_{j}+\text { age_group }_{k}+\text { lactation_group } \\
& +P C_{-} 1_{m}+P C \_2_{n}+e_{i j k l m n} .
\end{aligned}
$$

The first PC of this matrix aims to detect stratification at the breed level (Holstein vs. Brown Swiss), whereas the subsequent PC are expected to capture genomic differences between individuals. The first PC explained $43 \%$ of the variance, whereas the second PC explained $10 \%$.

The counterpart Bayesian model was performed to estimate the principal component effects (Sorensen and Gianola, 2002) using an in-home suite of programs written in $\mathrm{R}$ software ( $\mathrm{R}$ Core Team, 2015). Uniform distribution $U(-999,999)$ was assumed a priori for all the effects. Evidence of a host genetic effect was considered when $90 \%$ of the posterior distribution for the breed or the PC had the same sign (either positive or negative). This is, $90 \%$ of the posterior probability for the respective effect fell either above or below zero.

The estimated bacteria:archaea ratio from the $16 \mathrm{~S}$ rRNA region was 98:2. The more abundant bacterial phyla were Bacteroidetes (48\%), Firmicutes (32\%), and TM7 (Candidatus Saccharibacteria; 4\%). Methanobacteria were the most abundant clade among the archaeas. Taxa composition was similar to that reported before in other ruminal microbiome communities (Henderson et al., 2015; Wallace et al., 2015), being mainly microbes related to peptide and cellulose degradation or to the synthesis of microbial protein and VFA.

The RA of genera analyzed are shown in Figure 1. Prevotella was the most abundant bacteria-archaea genus in both breeds, followed by Butyrivibrio and Succiniclasticum. The archaea Methanobrevibacter was more abundant than the rest of the archaea genera detected in the samples.

Table 1 contains the results from the statistical analyses for the effect of the host genetic component on the different RA of bacteria and archaea. The PC2 showed differences for 9 (Methanobrevibacter, BS11 un- cultured, Prevotella, YRC22, S24-7 uncultured, 02d06, Butyrivibrio, Lachnospiraceae uncultured, and F16 uncultured) out of the 18 OTU genera analyzed from the $16 \mathrm{~S}$ rRNA region.

Figure 2 presents the RA of the analyzed ciliate in both breeds. The genus Isotrichia was the most abundant among the ciliate protozoal, followed by Entodinium (among the OTU that were classified into a known genus). Phenotypically, Isotricha and Entodinium were the most abundant known genera, followed by Ophryoscolex in Holsteins and Dasytricha in Brown Swiss. The genomic PC showed differences in 3 (Entodinium, Ophryoscolex, and Trichostomatia) out of 7 ciliate genera analyzed (Table 2).

Despite the small sample size, RA for $48 \%(12 / 25)$ of the genera analyzed were found to be associated with the genetic background of the animal, which suggests that the microbiome composition is regulated by some genetic mechanisms in the host. The genetic background was mainly detected by $\mathrm{PC} 2$ because it accounted for 2 Holstein groups and 1 Brown Swiss group; even after adjusting for PC1, which mainly accounted for breed effect. It seemed that $\mathrm{PC} 2$ was able to account for the breed plus some genetic background effect simultaneously (Supplemental Figure S1; https://doi.org/10 .3168/jds.2017-13179).

The host genetic background was associated with a similar proportion in both bacteria-archaea and ciliates ( $50 \%$ vs. $43 \%$ ). We did not find a host genetic effect on the RA of genera Dasytricha, Diplodinium, Isotricha, and Polyplastron. These microbes might be more influenced by diet than by the host genetic effect, and larger sample sizes might be necessary to detect differences for the host genetic effect.

Goodrich et al. (2016) also reported host genetic effects on the RA of different genera and families of Firmicutes and Euryarchaeota (e.g., Turicibacter, Blautia Clostridiaceae, Ruminococcaceae, Methanobrevibacter) in humans. This study also showed a host genetic effect on Tenericutes, Proteobacteria (family Oxalobacteraceae), and Actinobacteria (genera Bifidobacterium and Actinomyces). Our study also showed a host genetic effect on some genera of Firmicutes but also on some Bacteroidetes, contrary to their study, and ciliates, which were not analyzed in the human study because they are not abundant in the human gut. Roehe et al. (2016) estimated differences in the microbial community of progeny daughters from different cattle breeds and sires, suggesting that even under the same diet and environmental circumstances, individuals can differ in their microbial communities depending on the host genetic background.

Microbial networks for $16 \mathrm{~S}$ and $18 \mathrm{~S}$ gene rRNA regions were constructed using the network analysis 
(Cuscó et al., 2016), and their graphical representations are presented in Supplemental Figures S2 and S3 (https://doi.org/10.3168/jds.2017-13179). The microorganisms that showed to be related to the host genetics are relevant in the composition of the ruminal environment and the degradation of feed. For instance, bacteria from the genera Prevotella, the most abundant group, had a strong relationship with bacteria belonging to the Lachnospiraceae family in Supplemental Figure S2 (https://doi.org/10.3168/jds.2017-13179), suggesting that the increase in RA is related to the de- crease of microbes clustered in the Firmicutes phylum and the Lachnospiraceace family. The Prevotella and Paraprevotella genera are involved in the metabolism of proteins and peptides in the rumen. They break down protein and carbohydrates in feed (Jouany, 1991), synthesize de novo peptides, and use products of cellulose degradation from other cellulolytic bacteria (Lou et al., 1997; Atasoglu et al., 1998).

Another relationship that turns out to be interesting is the one detected between the Butyrivibrio and Prevotella genera. Some specific bacteria belonging to both

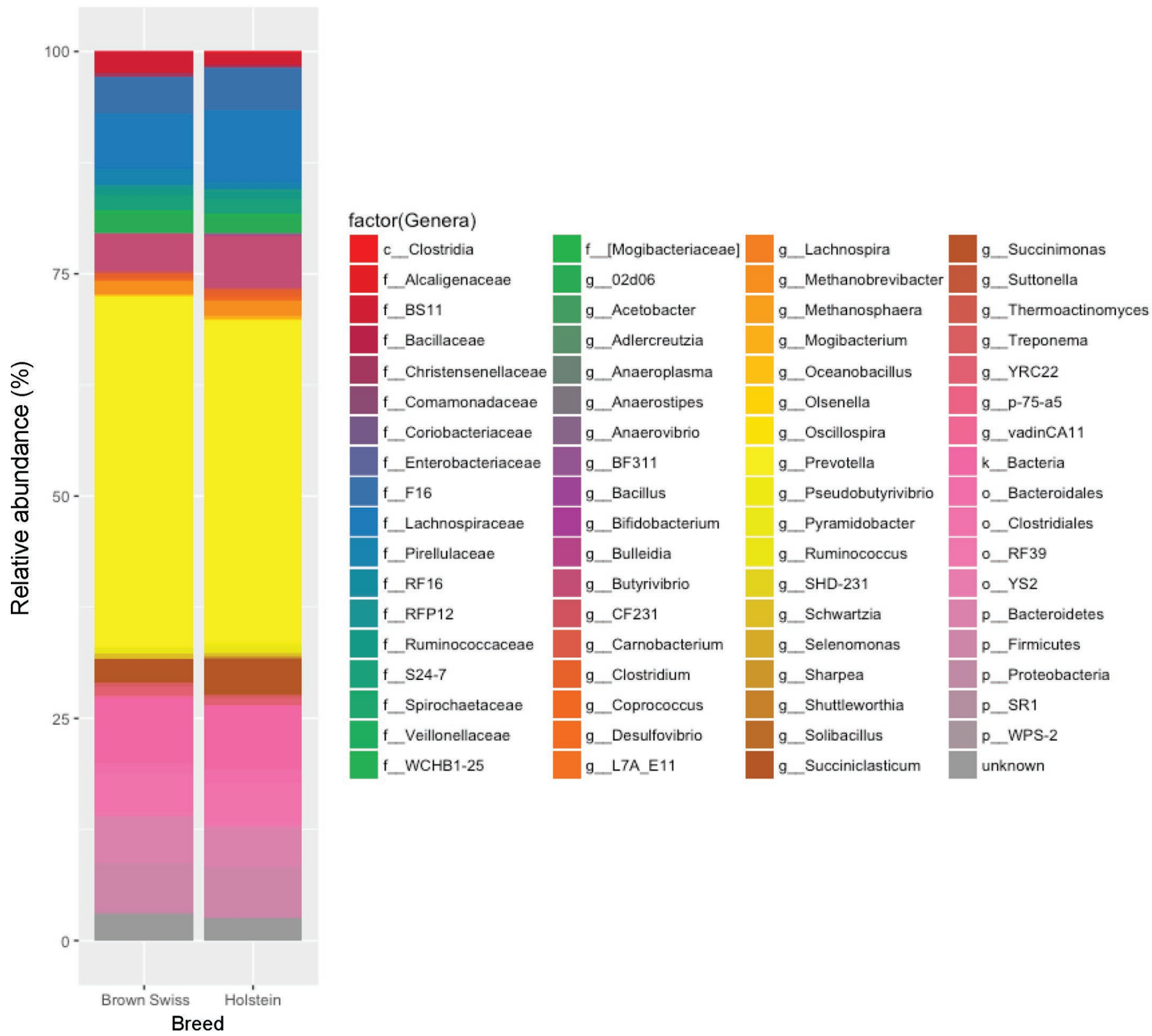

Figure 1. Rumen bacteria and euryarchaea composition. Relative abundance of bacteria and euryarchaea with average relative abundance $>0.1 \%$ in both breeds. Color version available online. 
genera are related to lipid metabolism, mainly with different steps of the ruminal biohydrogenation pathway of dietary UFA (Huws et al., 2011). Butyrivibrio bacteria are proteolytic bacteria and are involved in the degradation of hemicellulose walls. They produce mainly butyrate that is metabolized through the rumen wall to produce energy. In this sense, the Butyrivibrio genus shares an association with microbial species included in the Ruminococcaceae taxon (Supplemental Figure S2; https://doi.org/10.3168/jds.2017-13179). Further, bacteria from some genera, such as Ruminococcus, break down cellulose and hemicellulose and produce succinic acid as a major fermentation product together with acetic and formic acids, $\mathrm{H}_{2}$, and $\mathrm{CO}_{2}$. These products are then used by other bacteria, some from the Succiniclasticum genus, which convert succinate to propionate as an energy-yielding mechanism.

Archaea from the Methanobrevibacter genus use $\mathrm{H}_{2}$ and $\mathrm{CO}_{2}$ products and by-products from other microorganism (e.g., Ruminococcus) to synthesize methane. The archaea kingdom, mainly organisms related to the genera Methanobrevibacter and Methanosphaera, are highly associated with methane emission in ruminants (Jouany, 1991). Methanobrevibacter has been associated with methane emissions in many previous studies (Zhou et al., 2009, 2010; Wallace et al., 2015). On the other hand, the strong microbial association observed with the latter genus and Mogibacterium is noteworthy. Although most species have been isolated from human oral cavities and infected root canals, a recent study by Zhan et al. (2017) reported a linear reduction of its percentage with increasing dietary supplementation of alfalfa flavonoid extract in dairy cattle. Flavonoid compounds are used as a strategy to reduce ruminal methane production due to the decrease of a hydrogenotrophic methanogenic archaea population, which includes the Methanobrevibacter genus (Seradj et al., 2014).

Ciliate protozoal communities seem to have a relevant function in the maintenance of the ruminal ecosystem, as the most abundant genera (Dasytricha, Diplodinium, Entodinium, Eremoplastron, Isostricyha, and Trichostomatia) share strong interrelationships (Supplemental Figure S3; https://doi.org/10.3168/jds.2017-13179). Mainly, holotrich protozoa play a role in digestion and fermentation of feed components, in ruminal $\mathrm{O}_{2}$ utilization, and in the production of fermentation end products utilized by the host and $\mathrm{H}_{2}$ utilized by methanogenic bacteria (Lloyd et al., 1989; Williams and Colleman, 1992; Newbold et al., 2015). Entodinium ciliate are able to engulf small plant particles and degrade cellulose (Dauvrin, 1988; Jouany, 1991). They are considered as cellulolytic microorganisms. Isotricha and Dasytricha use soluble sugar, and many carbohydrate enzymatic activities have been detected. Polypastron ciliates can actively ingest large cellulosic fibers of the rumen fluid (Jouany, 1991; Belanche et al., 2012). The products of rumen ciliates are more or less similar and include acetate, butyrate, and lactate. They also produce $\mathrm{CO}_{2}$ and $\mathrm{H}_{2}$ during the synthesis that can be converted to methane by methanogenic archaea and protozoa. Ciliates interact with other rumen microorganism as they

Table 1. Effect of breed (Holstein vs. Brown Swiss) and the first 2 principal components (PC) of a genomic relationship matrix based on genotypes on the relative abundance of different rumen bacteria and archaea genera ${ }^{1}$

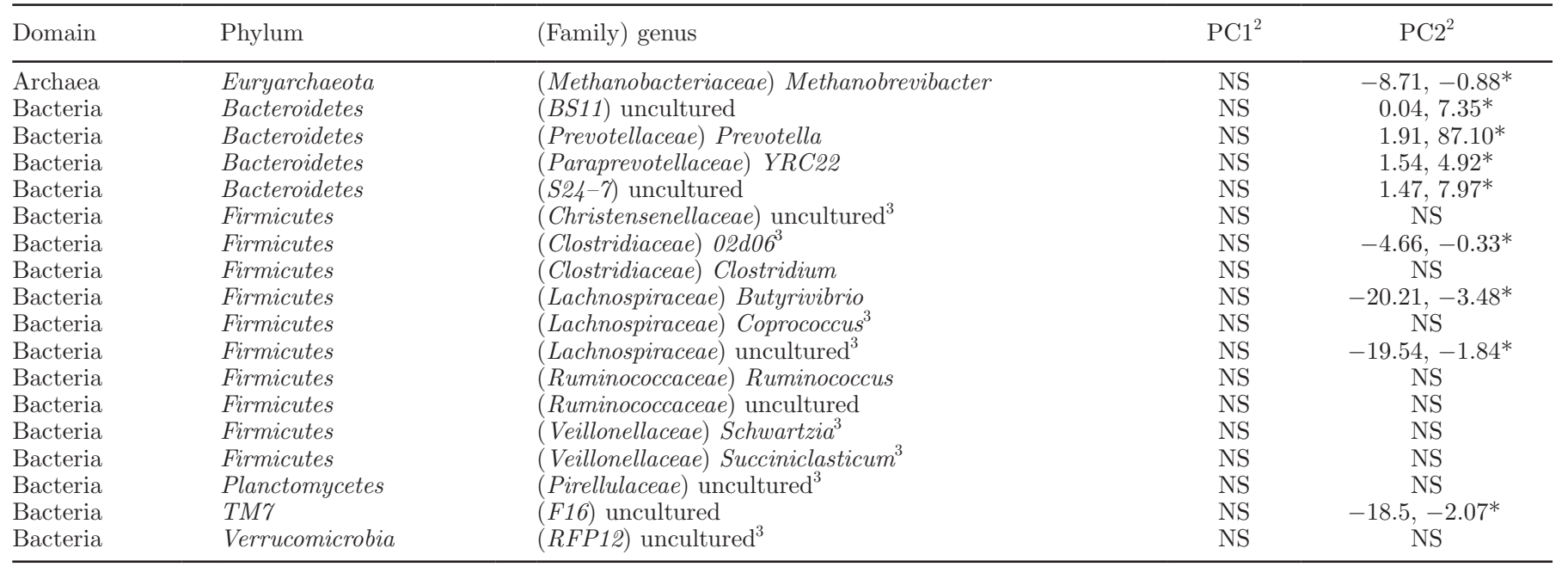

${ }^{1}$ Only genera that are present with average relative abundance $>0.1 \%$ in both breeds are shown.

${ }^{2}$ Asterisk $(*)$ indicates that $>90 \%$ of the posterior distribution of the effect was either above or below zero, suggesting a significant effect of the breed or of the principal component on the relative abundance (1-tailed test). NS (not significant) states otherwise.

${ }^{3}$ Relative abundance of the microbe was transformed to the Naeperian logarithmic scale to approximate a normal distribution. 
can ingest bacteria as a protein source. A host genetic effect on the RA of these microorganisms can partially explain the heritability found in related traits such as feed efficiency or methane yield (Basarab et al., 2013; Pryce et al., 2014, 2015).
The results of this study provide some evidence that supports the hypothesis of a host genetic component that can partially regulate the composition of the microbiome and indirectly some metabolic pathways. In this sense, it seems that there is a genetic component

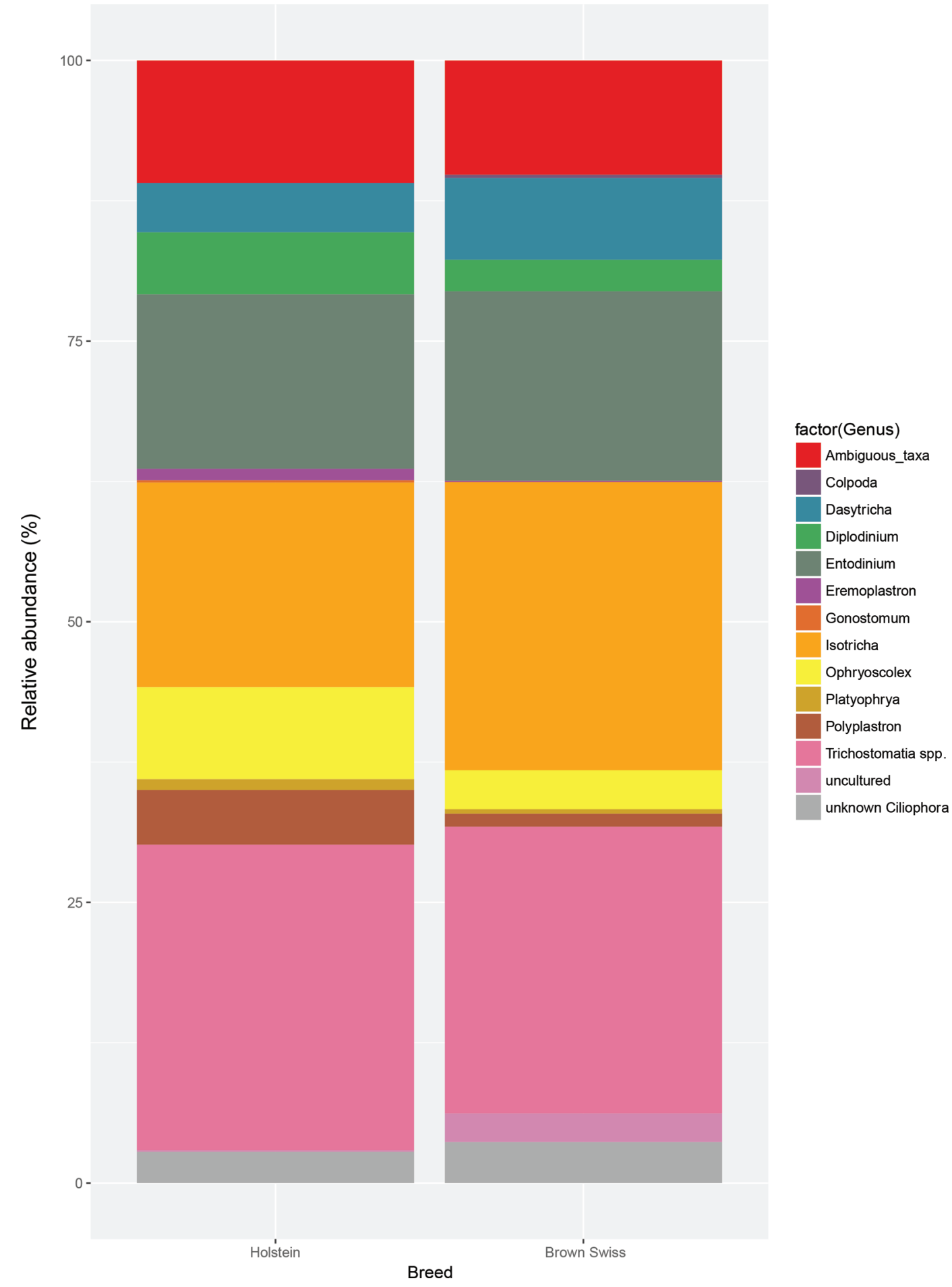

Figure 2. Rumen ciliate composition. Relative abundance of genera of ciliate with average relative abundance $>0.1 \%$ in both breeds. Color version available online. 
Table 2. Effect of breed (Holstein vs. Brown Swiss) and the first 2 principal components (PC) of a genomic relationship matrix based on genotypes on the relative abundance of different ciliate genera ${ }^{1}$

\begin{tabular}{|c|c|c|c|c|}
\hline Domain & Order & (Family) genus & $\mathrm{PC}^{2}$ & $\mathrm{PC} 2^{2}$ \\
\hline Eukaryota & Ciliophora & (Trichostomatia) Dasytricha & NS & NS \\
\hline Eukaryota & Ciliophora & (Trichostomatia) Diplodinium & NS & NS \\
\hline Eukaryota & Ciliophora & (Trichostomatia) Entodinium & NS & $0.11,58.13^{*}$ \\
\hline Eukaryota & Ciliophora & (Trichostomatia) Isotricha & NS & NS \\
\hline Eukaryota & Ciliophora & (Trichostomatia) Ophryoscolex ${ }^{3}$ & NS & $-14.52,-1.88^{*}$ \\
\hline Eukaryota & Ciliophora & (Trichostomatia) Polyplastron ${ }^{3}$ & NS & NS \\
\hline Eukaryota & Ciliophora & (Litostomatea) Trichostomatia & NS & $0.44,70.79 *$ \\
\hline
\end{tabular}

${ }^{1}$ Only genera that are present with average relative abundance $>0.1 \%$ in both breeds are shown.

${ }^{2}$ Asterisk $\left(^{*}\right)$ indicates that $>90 \%$ of the posterior distribution of the effect was either above or below zero, suggesting a significant effect of the breed or of the principal component on the relative abundance (1-tailed test). NS (not significant) states otherwise.

${ }^{3}$ Relative abundance of the microbe was transformed to the Naeperian logarithmic scale to approximate a normal distribution.

in the regulation of some groups of $\mathrm{H}_{2^{-}}$producing microorganisms included in the Firmicutes phylum and ciliate protozoa and $\mathrm{H}_{2}$-utilizing bacteria associated with Bacteroidetes. This is relevant because diets and management practices can be specifically designed to compensate those genotypes that are more susceptible to harboring less efficient microorganisms from a nutritional and energetic point of view. Breeding strategies can be applied to select future livestock generations prone to harboring a favorable microbiome composition that improves feed digestion and utilization while precluding the presence of harmful microbes or composition thereof. Results from this study must be considered carefully due to the reduced sample size, and future studies should allow researchers to better estimate the heritability of the microbiome composition in cattle as well as covariance components with other traits of interest (e.g., feed efficiency, productivity, or methane emissions).

\section{ACKNOWLEDGMENTS}

The authors thank Iurancha González (Neiker, Arkaute) for assistance during sampling and Beatriz Oporto (Neiker, Arkaute) and Medelin Ocejo (Neiker, Derio) for DNA preparations as well as the staff from Escuela Agraria de Fraisoro (Basque Department of Environment, Rural Development, Agriculture and Fishery; Zizurkil, Spain).

\section{REFERENCES}

Atasoglu, C., C. Valdés, N. D. Walker, C. J. Newbold, and R. J. Wallace. 1998. De novo synthesis of amino acids by the ruminal bacteria Prevotella bryantii B14, Selenomonas ruminantium HD4, and Streptococcus bovis ES1. Appl. Environ. Microbiol. 64:2836-2843.

Basarab, J. A., K. A. Beauchemin, V. S. Baron, K. H. Ominski, L. L. Guan, S. P. Miller, and J. J. Crowley. 2013. Reducing GHG emissions through genetic improvement for feed efficiency: Effects on economically important traits and enteric methane pro- duction. Animal 7(Suppl. 2):303-315. https://doi.org/10.1017/ S1751731113000888.

Belanche, A., G. de la Fuente, J. M. Moorby, and C. J. Newbold. 2012. Bacterial protein degradation by different rumen protozoal groups. J. Anim. Sci. 90:4495-4504. https://doi.org/10.2527/jas $.2012-5118$.

Caporaso, J. G., C. L. Lauber, W. A. Walters, D. Berg-Lyons, C. A. Lozupone, P. J. Turnbaugh, N. Fierer, and R. Knight. 2011. Global patterns of $16 \mathrm{~S}$ rRNA diversity at a depth of millions of sequences per sample. Proc. Natl. Acad. Sci. USA 108(Suppl. 1):4516-4522. https://doi.org/10.1073/pnas.1000080107.

Cuscó, A., J. Casellas, and O. Francino. 2016. CoMINO v.1.2: Network analysis program for directional interactions in microbiome. In The Barcelona Debates on the Human Microbiome: From Microbes to Medicines. International Center for Scientific Debates, Barcelona, Spain.

Dauvrin, T. 1988. La caractérisation de l'invertase du Cilié du rumen Isotricha prostoma, révèle certaines propriétés originales. Université Catholique de Louvain, Louvain-la-Neuve, Belgium.

Deng, W., D. Xi, H. Mao, and M. Wanapat. 2008. The use of molecular techniques based on ribosomal RNA and DNA for rumen microbial ecosystem studies: A review. Mol. Biol. Rep. 35:265-274. https://doi.org/10.1007/s11033-007-9079-1.

Goodrich, J. K., E. R. Davenport, M. Beaumont, M. A. Jackson, R. Knight, C. Ober, T. D. Spector, J. T. Bell, A. G. Clark, and R. E. Ley. 2016. Genetic determinants of the gut microbiome in UK twins. Cell Host Microbe 19:731-743. https://doi.org/10.1016/j .chom.2016.04.017.

Hayes, B. J., H. A. Lewin, and M. E. Goddard. 2013. The future of livestock breeding: Genomic selection for efficiency, reduced emissions intensity, and adaptation. Trends Genet. 29:206-214. https://doi.org/10.1016/j.tig.2012.11.009.

Henderson, G., F. Cox, S. Ganesh, A. Jonker, W. Young, and P. H. Janssen. 2015. Rumen microbial community composition varies with diet and host, but a core microbiome is found across a wide geographical range. Sci. Rep. 5:14567. https://doi.org/10.1038/ srep14567.

Huttenhower, C., R. Knight, C. T. Brown, J. G. Caporaso, J. C. Clemente, D. Gevers, E. A. Franzosa, S. T. Kelley, D. Knights, R. E. Ley, A. Mahurkar, J. Ravel, and O. White. 2014. Advancing the microbiome research community. Cell 159:227-230. https://doi .org/10.1016/j.cell.2014.09.022.

Huws, S. A., E. J. Kim, M. R. Lee, M. B. Scott, J. K. Tweed, E. Pinloche, R. J. Wallace, and N. D. Scollan. 2011. As yet uncultured bacteria phylogenetically classified as Prevotella, Lachnospiraceae incertae sedis and unclassified Bacteroidales, Clostridiales and Ruminococcaceae may play a predominant role in ruminal biohydrogenation. Environ. Microbiol. 13:1500-1512.

Jewell, K. A., C. A. McCormick, C. L. Odt, P. J. Weimer, and G. Suen. 2015. Ruminal bacterial community composition in dairy 
cows is dynamic over the course of two lactations and correlates with feed efficiency. Appl. Environ. Microbiol. 81:4697-4710. https://doi.org/10.1128/AEM.00720-15.

Jouany, J. P. 1991. Rumen Microbial Metabolism and Ruminant Digestion. Editions Quae, Versailles, France.

Kozich, J. J., S. L. Westcott, N. T. Baxter, S. K. Highlander, and P. D. Schloss. 2013. Development of a dual-index sequencing strategy and curation pipeline for analyzing amplicon sequence data on the MiSeq Illumina sequencing platform. Appl. Environ. Microbiol. 79:5112-5120. https://doi.org/10.1128/AEM.01043-13.

Lloyd, D., K. Hillman, N. Yarlett, and A. G. Williams. 1989. Hydrogen production by rumen holotrich protozoa: Effects of oxygen and implications for metabolic control by in situ conditions. J. Protozool. 36:205-213.

Lou, J., K. A. Dawson, and H. J. Strobel. 1997. Glycogen formation by the ruminal bacterium Prevotella ruminicola. Appl. Environ. Microbiol. 63:1483-1488.

Malmuthuge, N., and L. L. Guan. 2016. Gut microbiome and omics: A new definition to ruminant production and health. Anim. Front. 6:8-12. https://doi.org/10.2527/af.2016-0017.

Mohammed, R., G. E. Brink, D. M. Stevenson, A. P. Neumann, K. A. Beauchemin, G. Suen, and P. J. Weimer. 2014. Bacterial communities in the rumen of Holstein heifers differ when fed orchardgrass as pasture vs. hay. Front. Microbiol. 5:689. https://doi.org/ 10.3389/fmicb.2014.00689.

Newbold, C. J., G. de la Fuente, A. Belanche, E. Ramos-Morales, and N. McEwan. 2015. The role of ciliate protozoa in the rumen. Front. Microbiol. 6:1313 https://doi.org/10.3389/fmicb.2015.01313.

Pryce, J. E., O. Gonzalez-Recio, G. Nieuwhof, W. J. Wales, M. P. Coffey, B. J. Hayes, and M. E. Goddard. 2015. Hot topic: Definition and implementation of a breeding value for feed efficiency in dairy cows. J. Dairy Sci. 98:7340-7350. https://doi.org/10.3168/ jds.2015-9621.

Pryce, J. E., W. J. Wales, Y. de Haas, R. F. Veerkamp, and B. J. Hayes. 2014. Genomic selection for feed efficiency in dairy cattle. Animal 8:1-10. https://doi.org/10.1017/S1751731113001687.

R Core Team. 2015. R: A Language and Environment for Statistical Computing. R Foundation for Statistical Computing, Vienna, Austria. https://www.R-project.org/.

Roehe, R., R. J. Dewhurst, C.-A. Duthie, J. A. Rooke, N. McKain, D. W. Ross, J. J. Hyslop, A. Waterhouse, T. C. Freeman, M. Watson, and R. J. Wallace. 2016. Bovine host genetic variation influences rumen microbial methane production with best selection criterion for low methane emitting and efficiently feed converting hosts based on metagenomic gene abundance. PLoS Genet. 12:e1005846. https://doi.org/10.1371/journal.pgen.1005846.

Ross, E. M., P. J. Moate, L. C. Marett, B. G. Cocks, and B. J. Hayes. 2013. Metagenomic predictions: From microbiome to complex health and environmental phenotypes in humans and cattle. PLoS One 8:e73056. https://doi.org/10.1371/journal.pone.0073056.

Saro, C., M. J. Ranilla, and M. D. Carro. 2012. Postprandial changes of fiber-degrading microbes in the rumen of sheep fed diets varying in type of forage as monitored by real-time PCR and automated ribosomal intergenic spacer analysis. J. Anim. Sci. 90:4487-4494. https://doi.org/10.2527/jas.2012-5265.

Saro, C., M. J. Ranilla, M. L. Tejido, and M. D. Carro. 2014. Influence of forage type in the diet of sheep on rumen microbiota and fermentation characteristics. Livest. Sci. 160:52-59. https://doi.org/ 10.1016/j.livsci.2013.12.005.

Schloss, P. D., S. L. Westcott, T. Ryabin, J. R. Hall, M. Hartmann, E. B. Hollister, R. A. Lesniewski, B. B. Oakley, D. H. Parks, C. J. Robinson, J. W. Sahl, B. Stres, G. G. Thallinger, D. J. Van Horn, and C. F. Weber. 2009. Introducing mothur: Open-source, platform-independent, community-supported software for describing and comparing microbial communities. Appl. Environ. Microbiol. 75:7537-7541. https://doi.org/10.1128/AEM.01541-09.
Seradj, A. R., L. Abecia, J. Crespo, M. Fondevilla, and D. Villalba. 2014. The effect of Bioflavex and its pure flavonoid components on in vitro fermentation parameters and methane production in rumen fluid from steers given high concentrate diets. Anim. Feed Sci. Technol. 197:85-91.

Sorensen, D., and D. Gianola. 2002. Likelihood, Bayesian and MCMC Methods in Quantitative Genetics. Springer-Verlag, Berlin, Germany.

Wadhwa, M., M. P. S. Bakshi, and H. P. S. Makkar. 2016. Modifying gut microbiomes in large ruminants: Opportunities in non-intensive husbandry systems. Anim. Front. 6:27-36. https://doi.org/10 .2527 /af.2016-0020.

Waldor, M. K., G. Tyson, E. Borenstein, H. Ochman, A. Moeller, B B. Finlay, H. H. Kong, J. I. Gordon, K. E. Nelson, K. Dabbagh, and H. Smith. 2015. Where next for microbiome research? PLoS Biol. 13:e1002050. https://doi.org/10.1371/journal.pbio.1002050.

Wallace, R. J., J. A. Rooke, N. McKain, C.-A. Duthie, J. J. Hyslop, D. W. Ross, A. Waterhouse, M. Watson, and R. Roehe. 2015. The rumen microbial metagenome associated with high methane production in cattle. BMC Genomics 16:839. https://doi.org/10 .1186/s12864-015-2032-0.

Wang, L., Q. Xu, F. Kong, Y. Yang, D. Wu, S. Mishra, and Y. Li. 2016. Exploring the goat rumen microbiome from seven days to two years. PLoS One 11:e0154354. https://doi.org/10.1371/ journal.pone.0154354.

Wang, Q., G. M. Garrity, J. M. Tiedje, and J. R. Cole. 2007. Naive Bayesian classifier for rapid assignment of rRNA sequences into the new bacterial taxonomy. Appl. Environ. Microbiol. 73:52615267. https://doi.org/10.1128/AEM.00062-07.

Weimer, P. J., D. M. Stevenson, H. C. Mantovani, and S. L. C. Man. 2010. Host specificity of the ruminal bacterial community in the dairy cow following near-total exchange of ruminal contents. J. Dairy Sci. 93:5902-5912. https://doi.org/10.3168/jds.2010-3500.

Williams, A. G., and G. S. Coleman. 1992. The Rumen Protozoa. Springer-Verlag, New York, NY.

Yáñez-Ruiz, D. R., B. Macías, E. Pinloche, and C. J. Newbold. 2010. The persistence of bacterial and methanogenic archaeal communities residing in the rumen of young lambs. FEMS Microbiol. Ecol. 72:272-278. https://doi.org/10.1111/j.1574-6941.2010.00852.x.

Yang, J., T. A. Manolio, L. R. Pasquale, E. Boerwinkle, N. Caporaso, J. M. Cunningham, M. de Andrade, B. Feenstra, E. Feingold, M. G. Hayes, W. G. Hill, M. T. Landi, A. Alonso, G. Lettre, P. Lin, H. Ling, W. Lowe, R. A. Mathias, M. Melbye, E. Pugh, M. C. Cornelis, B. S. Weir, M. E. Goddard, and P. M. Visscher. 2011. Genome partitioning of genetic variation for complex traits using common SNPs. Nat. Genet. 43:519-525. https://doi.org/10.1038/ng.823.

$\mathrm{Yu}, \mathrm{Z}$., and R. Foster. 2005. Methods in Gut Microbial Ecology for Ruminants. Springer Netherlands, Dordrecht, the Netherlands.

Zhan, J., M. Liu, C. Wu, X. Su, K. Zhan, and G. Zhao. 2017. Effects of alfalfa flavonoids extract on the microbial flora of dairy cow rumen. Asian-Australas. J. Anim. Sci. 30:1261-1269. https://doi .org/10.5713/ajas.16.0839.

Zhang, Y., W. Gao, and Q. Meng. 2007. Fermentation of plant cell walls by ruminal bacteria, protozoa and fungi and their interaction with fibre particle size. Arch. Anim. Nutr. 61:114-125. https://doi .org/10.1080/17450390701204020.

Zhou, M., E. Hernandez-Sanabria, and L. L. Guan. 2009. Assessment of the microbial ecology of ruminal methanogens in cattle with different feed efficiencies. Appl. Environ. Microbiol. 75:6524-6533. https://doi.org/10.1128/AEM.02815-08.

Zhou, M., E. Hernandez-Sanabria, and L. L. Guan. 2010. Characterization of variation in rumen methanogenic communities under different dietary and host feed efficiency conditions, as determined by PCR-denaturing gradient gel electrophoresis analysis. Appl. Environ. Microbiol. 76:3776-3786. https://doi.org/10.1128/AEM $.00010-10$. 